\title{
Editorial - Practice of Arthroscopy, is it any different in India?
}

\author{
David V. Rajan ${ }^{1}$
}

(C) Indian Orthopaedics Association 2021

Welcome to this special edition on Arthroscopy for the Indian Journal of Orthopaedics. We have come a long way since the inception of Arthroscopic surgery in our country when introduced by stalwarts like Bob Jackson, Dinesh Patel and Gopal Krishnan as early as 1985.

Back in the day, trauma surgeries, joint replacement and deformity corrections were prioritised over quality of life inducing arthroscopic surgery. Hence, the prevailing practice of Arthroscopy in India during former times was dissimilar to that in developed countries owing to subjective reasons specific to our country: cost constraints, lack of public awareness and import restrictions. Fast forward to the present and Indian arthroscopists are on par with their international counterparts' apropos academics, research and surgical skills. The current edition bears testimony to the emergence of India as torchbearer in the golden era of arthroscopic practice [1].

This edition contains high-quality reviews and original articles encompassing all limb-specific specialities. Popularly cited researchers worldwide, exposed to their Indian counterparts through varied interactions have contributed through distilling their valuable experiences into print. The intention of this edition is to enlighten and adequately inform young orthopaedic surgeons interested in the subspeciality of arthroscopy and sports medicine: offer direction in which he/she can strive for. The cognising of limitations and challenges in replicating every procedure performed in developed/first world nations cannot be stressed enough. We have endeavoured in this special edition to offer young upcoming surgeons, the various options available in arthroscopic surgery with the intent to redirect focus in their career or at the least create awareness.

This special edition also contains articles on knee, shoulder, ankle and elbow arthroscopy as well as related

David V. Rajan

davidvrajan@gmail.com

1 Department of Arthroscopy and Sports Medicine, Ortho One-Orthopaedic Speciality Centre, Coimbatore, India surgeries. We have requested the authors to provide reviews and expound on medical conditions locally prevalent and hence of greater relevance. Post-reconstructive infections are common due to the challenging environment and this has been dealt with through offering valuable guidelines in case of post-op infection.

Articles on shoulder by Prof. Khalid offer valuable insight on the relevance of open cuff repair and explains how, why and when we can perform open rotator cuff repairs. The article "Evolving concept on shoulder instability management" by Prof. Nikhil Verma and his colleagues, is an outstanding guide to treatment of instability with evidence gleaned from current literature. The much-fancied Latarjet procedure is a must-have in a shoulder surgeon's armamentarium and avoids the need for cost-prohibitive implants, a technical note on this procedure is also included in this edition.

An exhaustive and well-illustrated review on clinical guidelines in the management of periarthritis shoulder has been presented by Dr Vivek Pandey.

The review on current concepts in tennis elbow by Prof. Jonathan Herald and colleagues discusses surgical management of recalcitrant lateral epicondylitis, emphasising knotless suture anchors. Ulnar-sided wrist pain is often overlooked, hence an algorithm of this condition with emphasis on its management has been documented by Dr Darshan Kumar and Dr Abhijeet Wahegaonkar.

Though booming world over, hip arthroscopy is fairly uncommon in India. Hip-related issues like labral tears, etc. are either uncommon or the pain threshold sufficiently high amongst the Indian populace causing their neglect. The article by Prof Lalit Maini and his colleagues pertaining to hip arthroscopy's infrequency in India is well dealt with.

The review articles on meniscal root repair techniques and revision ACL reconstruction are also covered in this edition. An attempt at understanding the healing process following repair of bucket-handle meniscal tears using indirect MR arthrography is described by Dr Sachin Tapasvi and his colleagues. 
The role of orthobiologics in sports medicine has immense potential, and its value in areas of interest is well elucidated by Dr Arumugam S and his colleagues.

Clinical practice guidelines on chondral lesions are well recounted in the paper by Dr Sumit Banerjee and his colleagues.

"Limit yourself to what is needed"- the art of minimalism and economy needs to be practiced with the single objective of patient's need when weighing against all available options [2].

To conclude: this edition emphasises and focusses on how arthroscopic practice may be rendered relevant in the larger Indian sub-context. A minimalistic approach without compromising on surgical safety and quality is not only desirable but also imperative. Specific articles and topics related to arthroscopic practice have been handpicked and delivered by pioneers in the field. The collection of articles and guidelines to follow will aid arthroscopists in the efficacious employment of their surgical acumen availing evidence-based medical practices rather than intuition.

\section{Compliance with ethical standards}

Conflict of interest The authors declare that they have no conflict of interest.

Ethical standard statement This article does not contain any studies with human or animal subjects performed by the any of the authors.

Informed consent For this type of study informed consent is not required.

\section{References}

1. Rajan, D. V., Ashraf, M., Challumuri, N., \& Sahanand, S. K. (2020). History of arthroscopy in India: Origins and evolution. Journal of Arthroscopic Surgery and Sports Medicine., 1(1), $5-10$.

2. Van Dijk, C. N. (2017). JISAKOS, 2, 121-122. https://doi. org/10.1136/jisakos-2017-000148. 